regions of the molecular surface that are involved in ligand recognition ${ }^{3}$. It is energetically favourable for hydrophobic amino-acid side chains in globular proteins to associate to form a hydrophobic core, a process called hydrophobic collapse. Charged amino-acid side chains are excluded from the hydrophobic core and become exposed to solvent at the surface. For disordered proteins such as Pro-T $\alpha$ and H1, however, the high percentage of charged amino-acid residues precludes hydrophobic collapse, and the distribution of charges skews the conformational ensemble to a more expanded state than would be observed if the proteins were stably folded ${ }^{4}$, leaving the charged side chains fully exposed to the solvent.

Pro-T $\alpha$ and $\mathrm{H} 1$ become more compact when they bind to each other, probably because charge complementarity in disordered proteins can mediate the compaction of protein chains through electrostatic attractive forces, in addition to driving intermolecular interactions ${ }^{5}$. The authors find that the high degree of charge complementarity between Pro-Ta and $\mathrm{H} 1$ also provides a substantial electrostatic contribution to the binding energy of the system, making complex formation extremely favourable - as reflected by the high binding affinity of Pro-Ta for H1. The distribution of charges throughout the amino-acid sequences of both Pro-Ta and $\mathrm{H} 1$ allows the formation of a wide range of stable complexes that lack defined binding sites.

Many disordered proteins form 'fuzzy' complexes $^{6}$, which have a high degree of structural heterogeneity. Pro-Ta and H1 form an archetypal fuzzy complex that involves a large ensemble of possible bound protein conformations, many of which are adopted by only a small number of individual complexes and occur with approximately equal probability. The rate of association of Pro-Ta and $\mathrm{H} 1$ is limited by the diffusion of the molecules, a hallmark of electrostatic attraction ${ }^{7}$. The rapid association, slow dissociation and broad distribution of charge throughout the Pro-T $\alpha$ and $\mathrm{H} 1$ sequences are responsible for the formation of the heterogeneous ensemble of complexes, in which the proteins entwine in many different configurations (Fig. 1).

The interaction mechanism of Pro-T $\alpha$ and $\mathrm{H} 1$ probably aids their biological function. Pro-Ta assists with the assembly and disassembly of chromatin, the material in which DNA is packaged with histone proteins (such as $\mathrm{H} 1$ ) in cells ${ }^{8}$. To perform its function, Pro-Ta must recognize its histone substrates rapidly and with sufficient affinity to compete with the high affinity of histone-DNA interactions. The high binding affinity of Pro-Ta for $\mathrm{H} 1$ and the association rate of the two proteins imply that the dissociation of Pro-Ta-H1 complexes is slow enough to allow functional outcomes, but fast enough not to slow down biological turnover.

Many mechanistic questions remain. Can
Pro-Ta form complexes with chromatin-bound $\mathrm{H} 1$, to promote the dissociation of $\mathrm{H} 1$ from DNA and to usher it to a new binding site, without having to wait for spontaneous dissociation? Similarly, can Pro-Ta remain bound to $\mathrm{H} 1$ once it has been deposited at a new DNA site? The flexibility in the Pro-Ta-H1 complexes would facilitate such processes: the positively charged regions of $\mathrm{H} 1$ would be exposed even when in complex with Pro-Ta, and thus be available for simultaneous binding to chromatin.

It is evident that the amino-acid sequences of Pro-Ta and $\mathrm{H} 1$ have a crucial role in dictating the proteins' molecular function. The amino-acid sequences of disordered regions in proteins evolve rapidly, yet recent studies have shown that the net charge is conserved despite a high degree of sequence diversity (see ref. 9, for example). Highly charged proteins such as Pro-Ta and H1 might therefore be more tolerant to mutation than their less-charged counterparts. As noted by Borgia and colleagues, many disordered proteins have levels of net charge similar to those of Pro-T $\alpha$ and $\mathrm{H} 1$, suggesting that the formation of dynamic complexes between disordered proteins of opposite charge might be common.

Charge complementarity between disordered proteins and their molecular partners is of great importance to signalling pathways that rely on post-translational modifications (protein modifications that occur after protein biosynthesis), and to phase-separation processes that result in the formation of concentrated droplets of proteins and nucleic acids ${ }^{10}$. Although it has long been evident that electrostatic interactions have a central role in the formation of biological complexes, Borgia and colleagues' work highlights how crucial these attractive forces can be for the assembly of very strong, yet highly dynamic, molecular complexes in the cell.

Rebecca B. Berlow and Peter E. Wright are in the Department of Integrative Structural and Computational Biology and The Skaggs Institute of Chemical Biology, The Scripps Research Institute, La Jolla, California 92037, USA.

e-mail:wright@scripps.edu

1. Borgia, A. et al. Nature 555, 61-66 (2018).

2. Mollica, L. et al. Front. Mol. Biosci. 3, 52 (2016).

3. DeLano, W. L. Curr. Opin. Struct. Biol. 12, 14-20 (2002).

4. Mao, A. H., Crick, S. L., Vitalis, A., Chicoine, C. L. \& Pappu, R. V. Proc. Natl Acad. Sci. USA 107, 8183-8188 (2010).

5. Mittag, T. et al. Proc. Natl Acad. Sci. USA 105, 17772-17777 (2008)

6. Tompa, P. \& Fuxreiter, M. Trends Biochem. Sci. 33, 2-8 (2007).

7. Zhou, H. X. \& Bates, P. A. Curr. Opin. Struct. Biol. 23, 887-893 (2013).

8. George, E. M. \& Brown, D. T. FEBS Lett. 584, 2833-2836 (2010).

9. Zarin, T., Tsai, C. N., Nguyen Ba, A. N. \& Moses, A. M. Proc. Natl Acad. Sci. USA 114, E1450-E1459 (2017).

10.Wright, P. E. \& Dyson, H. J. Nature Rev. Mol. Cell Biol. $16,18-29$ (2015).

This article was published online on 21 February 2018.

\title{
A surprising chill before the cosmic dawn
}

An experiment to estimate when stars began to form in the Universe suggests that gas temperatures just before stars appeared had fallen well below predicted limits, and that dark matter is not as shadowy as was thought. SEE LETTER P.67

\section{LINCOLN GREENHILL}

$\mathrm{T}$ The first stars to form generated copious fluxes of ultraviolet radiation that suffused the early Universe - a phenomenon referred to as the cosmic dawn. Many calculations have been performed to estimate when this occurred ${ }^{1}$, but no datadriven constraints on the timing have been available. On page 67, Bowman et al. ${ }^{2}$ report what might be the first detection of the thermal footprints of these stars, tracking back to 180 million years after the Big Bang.

Less than one million years after the Big Bang, the Universe consisted of atomic gas (chiefly hydrogen) and a form of matter that outweighs regular matter by more than five times $^{3}$ but has yet to be seen directly. Measurements over decades have indicated that, oddly enough, this 'dark' matter interacts with itself and with regular matter only through the action of gravity. It was mainly the gravity of dark matter that amplified small, localized density perturbations in the Universe shortly after the Big Bang to generate the first largescale structures. But it was the hydrogen within these perturbations that collapsed piecemeal to form stars, bringing about the cosmic dawn.

The observable thermal footprints of early stars derive from small variations in the ratio of the number of interstellar hydrogen atoms found in two particular energy states; 
a transition between these states causes a photon to be emitted or absorbed at a characteristic radio frequency. The ratio reflects the degree of excitation of the hydrogen, and can be expressed as a temperature, known as the atomic spin temperature $\left(T_{\mathrm{S}}\right)$.

At early times, when the Universe was relatively small and mean gas density was high, collisions between atoms were frequent. $T_{\mathrm{S}}$ was therefore the same as the kinetic temperature of the gas $\left(T_{\mathrm{G}}\right)$, an indicator of the energy available to excite atoms through collisions. By the time stars began to form, the Universe had expanded. Both $T_{\mathrm{G}}$ and mean gas densities had fallen, and collisions were infrequent, allowing $T_{\mathrm{S}}$ to drift upward to the temperature of the radiation $\left(T_{\mathrm{R}}\right)$ left over from the Big Bang (Fig. 1). $T_{\mathrm{R}}$ also fell as the Universe expanded, but not as quickly as $T_{\mathrm{G}}$.

A long-standing theor $y^{4,5}$ that still awaits testing predicts that absorption of UV radiation from early stars by nearby clouds of hydrogen could have driven $T_{\mathrm{S}}$ back down to $T_{\mathrm{G}}$, but not lower. In other words, the cosmic dawn would make the gas seem colder when observed at radio frequencies. This would create an absorption feature in the spectrum of the background radiation left over from the Big Bang.

Bowman et al. now report the possible detection of just such an absorption signal. The authors measured $T_{\mathrm{S}}$, averaged over much of the sky and over a contiguous range of radio frequencies; each frequency provides a window on a different time in the Universe's past. The measurement is very difficult because it must be performed using an extremely well-calibrated VHF radio antenna and receiver, to enable the weak cosmological signal to be separated from much stronger celestial signals and from those within the electronics systems of the apparatus used.

The putative absorption signal extends over a wide frequency range, one end of which looks as far back as 180 million years after the Big Bang, in good agreement with theoretical predictions ${ }^{6}$. Remarkably, however, the peak amplitude of the absorption is two to three times larger than predicted by the most optimistic models, and the absorption profile is flat-bottomed, rather than curvilinear and Gaussian-like, which is also at odds with models.

So how can the differences from the models be explained? On page 71, Barkana $^{7}$ argues that models could achieve the reported signal amplitude and profile if non-gravitational interactions - like those that occur between charged particles - occur between dark matter and normalmatter particles, and if the dark-matter particles

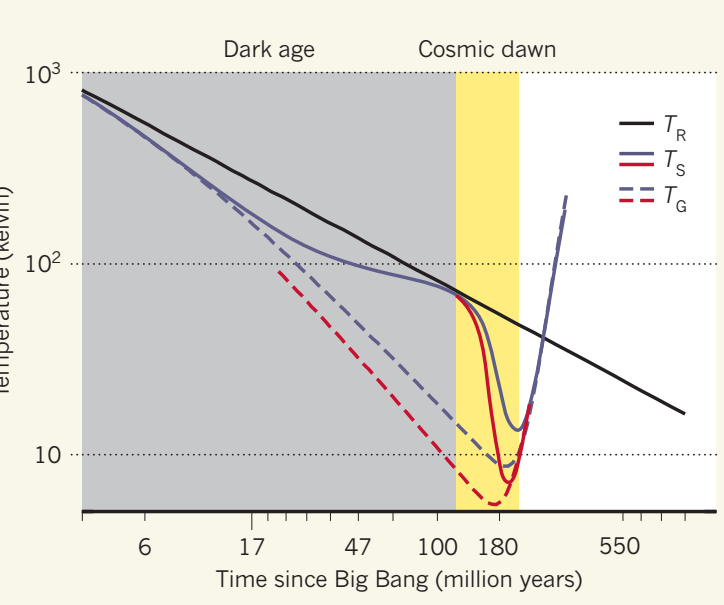

igure 1 | Temperature changes during the evolution of the niverse. The first two phases of the Universe were the 'dark age', before diation $\left(T_{\mathrm{R}}\right)$ left over from the Big Bang has declined slowly over time. The spin temperature $\left(T_{S}\right)$ of hydrogen that has not formed stars reflects the excitation state of the hydrogen atoms (solid blue line shows previous mate $T_{S}$, and find that it dropped to lower values (red solid line) than in models. Barkana proposes that this could be evidence a previously unrecognized, non-gravitational interaction between mermal and dark matter. Such interactions would mean that the 'kinetic' (red dashed line) than is predicted by known physics (blue dashed line). Adapted from ref. 12.)

have relatively low masses and velocities that are less than the speed of light. The effects of variously hypothesized types of dark matter have been calculated previously ${ }^{8,9}$, but only those in which dark matter and normal matter scatter each other increase the magnitude of the absorption signature. The idea that a detectable radio signal from the cosmic dawn can be connected to the particle properties of dark matter suggests a potentially revolutionary angle for exploring fundamental physics.

Bowman and colleagues' claim to have detected the long-sought absorption signal is bolstered by myriad tests in which the authors altered their experimental hardware or data analysis, in a concerted effort to identify systematic errors that might be responsible for the measured signal. The tests included repeating the data acquisition and analysis using a duplicate antenna at a second, nearby location; orienting the antenna at different angles with respect to the compass; and changing the ways in which the antenna is isolated from the ground. Other tests focused on switching various facets of the data calibration on and off.

However, the most stringent test will be to compare the current results with those to come from independent experiments also aimed at detecting the cosmic-dawn signal ${ }^{10,11}$. I hope that the unexpected amplitude and line shape of the reported absorption signal is indeed a hard-won breakthrough that reveals evidence of unexpected physics. But it is possible that systematic errors have escaped detection by the tests that were run. Two extensions to the reported tests include using circuitry that more precisely imitates the antenna than Bowman and colleagues' circuitry when attached to the receiver during performance evaluation and calibration, and the cross-checking of performance models for the antenna (which are currently based on computer simulations of antenna electromagnetics) with field measurements made when narrowband or sinusoidal signals are broadcast near the antenna.

Bowman and co-workers' report will be recognized as a milestone for this nascent experimental field: the first reputable claim of a muchanticipated detection. The followup will not be limited to ever finer interpretations of increasingly accurate one-dimensional spectra. Studies of the cosmic-dawn signal using interferometers (arrays of antennas) could describe the 3D structure of the Universe at that time and, by extrapolation, during the primordial 'dark age' when large-scale structure in the Universe first formed. One of Barkana's particularly notable predictions is that, if non-gravitational interactions between normal and dark matter do exist, then the absorption signal detectable by interferometers could be stronger and more distinctive than had been predicted. It would encode the spatial fluctuations of matter density that occurred during the dark age, rather than just gas temperature, thus presenting new opportunities for tests of fundamental physics.

Lincoln Greenhill is in the Radio and Geoastronomy Division, Smithsonian Astrophysical Observatory, Cambridge, Massachusetts 02138, USA, and in the Department of Astronomy, Harvard University, Cambridge.

e-mail: greenhill@cfa.harvard.edu

1. Pritchard, J. R. \& Loeb, A. Rep. Prog. Phys. $\mathbf{7 5}$ 086901-086935 (2012).

2. Bowman, J. D., Rogers, A. E. E., Monsalve, R. A., Mozdzen, T. J. \& Mahesh, N. Nature 555, 67-70 (2018).

3. Ade, P. A. R. et al. Astron. Astrophys. 571, A16 (2014)

4. Wouthuysen, S. A. Astrophys. J. 57, 31-32 (1952)

5. Field, G. B. Astrophys. J. 129, 536-550 (1959).

6. Cohen, A., Fialkov, A., Barkana, R. \& Lotem, M. Mon. Not. R. Astron. Soc. 472, 1915-1931 (2017)

7. Barkana, R. Nature 555, 71-74 (2018)

8. Evoli, C., Mesinger, A. \& Ferrara, A. J. Cosmol. Astropart. Phys. 024 (2014).

9. Tashiro, H., Kadota, K. \& Silk, J. Phys. Rev. D 90 , 083522 (2014).

10.Price, D. C. et al. Preprint at http:// arXiv: 1709.09313 (2017)

11.Singh, S. et al. Preprint at http://arXiv:1710.01101 (2017).

12.Pritchard, J. \& Loeb, A. Phys. Rev. D 78, 103511-6 (2008). 